\title{
Mechanism of coordination between cell and chloroplast division in unicellular algae
}

\author{
Nobuko Sumiya \\ Department of Biology, Keio University, Hiyoshi 4-1-1, Kohoku-ku, Yokohama, Kanagawa, 223-8521, Japan \\ Author for correspondence: N. Sumiya, sumiyan@keio.jp
}

\begin{abstract}
Summary: Chloroplasts arose from a cyanobacterial endosymbiont. Most algae with a single or a few chloroplasts per cell synchronize chloroplast division with the host cell cycle. This synchronization is regulated via interactions between the cell and chloroplast. The onset of chloroplast division is regulated by S-phase-specific expression of the nuclear-encoded chloroplast division genes; however, in the unicellular alga Nannochloris bacillaris, one of the plastid division genes, FtsZ2, is expressed throughout the cell cycle. Plastiddividing (PD) machinery usually forms a ring complex only during the division phase, although the number of the FtsZ rings, and not of PD rings, is increased in $N$. bacillaris cells under phosphate-enriched mixotrophic condition where the chloroplast DNA is excessively replicated. Therefore, among components of the PD machinery, only FtsZ ring formation is promoted by chloroplast DNA synthesis under certain conditions. During chloroplast division, the onset of chloroplast constriction allows the progression of cell cycle to metaphase. The blockage of PD machinery formation before its assembly arrests the cell cycle in prophase in the unicellular alga, Cyanidioschyzon merolae; however, once DRP5B is recruited to the chloroplast division site, the cell cycle progresses despite the failure of chloroplast fission. A similar phenomenon has been observed in the glaucophyte Cyanophora paradoxa suggesting that the mechanism of the chloroplast division checkpoint is established early in chloroplast acquisition.
\end{abstract}

Key words: cell cycle, chloroplast division, Cyanidioschyzon merolae, Cyanophora paradoxa, FtsZ, Nannochloris bacillaris

\section{INTRODUCTION}

Chloroplasts are believed to have arisen from the endosymbiosis of a cyanobacterium with a eukaryotic host cell. The presence of undigested chloroplasts, referred to as kleptochloroplasts, within the protist suggests that the coordination of host cell and cyanobacterial division is essential for the acquisition and inheritance of chloroplasts (Dorrell and Howe 2012, Pedroza-Garcia et al. 2016). For example, the flagellate Hatena arenicola harbors a chlorophyte alga, Nephroselmis, as a temporal symbiont. The division of $H$. arenicola and Nephroselmis is not synchronized; therefore, the Nephroselmis cell is inherited by only one of the two daughter cells (Okamoto and Inouye 2005, 2006). The dinoflagellate Nusuttodinium aeruginosum retains the chloroplast from the ingested cryptomonad prey (Onuma and Horiguchi 2015). The kleptochloroplast divides during the host cell division and is inherited by both daughter cells, however, the cryptomonad nucleus does not. The kleptochloroplast of the daughter cell that does not inherit the cryptomonad nucleus is digested after several cycles of host cell division. These studies indicate that the establishment of the coordinated division of the host cell and symbiont is crucial for the permanent acquisition of chloroplasts.

Chloroplasts are divided into two with the prokaryoticand eukaryotic-derived plastid-dividing (PD) machinery (Miyagishima 2011, Chen et al. 2018). Cyanobacterial-derived FtsZ and eukaryotic-derived dynamin-related protein DRP5B are well conserved among the algae and land plants. During chloroplast division, FtsZ initially assembles into a ring on the stromal side of the inner chloroplast membrane at the division site (Mori et al. 2001, Vitha et al. 2001, Kuroiwa et al. 2002). Thereafter, the plastid division ring is assembled on the stromal side of the inner membrane (inner PD ring) and on the cytosolic side of the outer membrane (outer PD ring composed of polyglucan nanofilaments) (Kuroiwa et al. 1998, Miyagishima et al. 1999, Yoshida et al. 2010). Eventually, DRP5B assembles on the cytosolic side of the outer chloroplast membrane and generates the motive force for the outer PD ring contraction, thereby pinching off the chloroplast (Figure 1; Gao et al. 2003, Miyagishima et al. 2003, Yoshida et al. 2006).

Land plants have multiple chloroplasts per cell, and chloroplast division is not coordinated with cell division (Miyagishima 2011, Pedroza-Garcia et al. 2016). Alternatively, because most unicellular algae contain a single or a few chloroplasts per cell, the mechanisms regulating the coordinated division of the cell and chloroplast are expected to exist. Studies investigating the relationship between the PD machinery formation and host cell cycle will help us understand the mechanism(s) involved in the permanent acquisition of chloroplasts. In this review, I introduce the interactive regulations in the division synchronization of the host cell and chloroplast in unicellular algae.

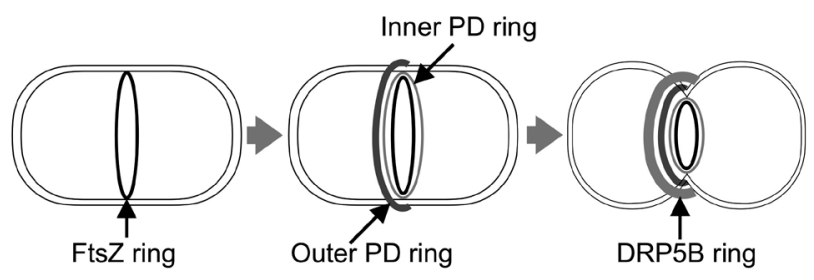

Figure 1 Working model of the plastid-dividing (PD) machinery during chloroplast division. FtsZ first assembles as a ring on the stromal side of the inner envelope. The inner and the outer PD rings are formed on the stromal side of the inner envelope and the cytosolic side of the outer envelope, respectively. After the assembly of the dynamin-related protein DRP5B on the cytosolic side of the outer envelope, the division site begins to constrict. 
FtsZ EXPRESSION AND RING FORMATION IN THE UNICELLULAR ALGA, Nanochloris Bacillaris

FtsZ is a structural homolog of tubulin and polymerizes into filaments, which generates a bending force on the membrane (Srinivasan et al. 2008, Erickson et al. 2010). In bacteria, FtsZ recruits other cell division proteins and cell wall synthesis machinery to induce cell division (Bisson-Filho et al. 2017, Yang et al. 2017). In green plants, two FtsZ homologs, FtsZ1 and FtsZ2, have been isolated. To understand the difference between the two homologs, the expression patterns of FtsZ1 and FtsZ2 have been examined in the unicellular alga, Nannochloris bacillaris (Trebouxiophyceae). N. bacillaris contains a single chloroplast and proliferates by binary fission (Arai et al. 1998, Yamamoto et al. 2001, 2003, 2007). Two FtsZ paralogues (NbFtsZ1 and NbFtsZ2) are isolated and they are grouped into the FtsZ1 and FtsZ2 families of green plants (Koide et al. 2004). Approximately, $80 \%$ of $N$. bacillaris cells divide synchronously in an inorganic medium under a $12 \mathrm{~h}$ light/ $12 \mathrm{~h}$ dark photoperiod (Sumiya et al. 2012a). In synchronous culture, NbFtsZ1 is expressed only during chloroplast division phase, whereas $N b F t s Z 2$ is expressed throughout the cell cycle (Sumiya et al. 2012a). NbFtsZ1 and NbFtsZ2 expression level are further compared between the autotrophic and mixotrophic conditions. The proportion of dividing cells under mixotrophic condition is approximately two times higher than that under autotrophic condition. Accordingly, the expression of NbFtsZ1 under mixotrophic condition is twice as high as the expression under autotrophic condition. In contrast, the expression of NbFtsZ2 under mixotrophic condition is 8-fold higher than that under autotrophic condition, suggesting that the expression of $\mathrm{NbFtsZ2}$ is not regulated by the cell cycle but by other factors, such as medium conditions (Sumiya et al. 2012a).
Immunofluorescence detection of NbFtsZ using anti-NbFtsZ antibody reveals that NbFtsZ assembles as a ring at the chloroplast division site only during chloroplast division under autotrophic condition, but throughout the cell cycle under mixotrophic condition (Fig. 2B; Sumiya et al. 2012a). Although the anti-NbFtsZ antibody failed to differentiate between NbFtsZ1 and NbFtsZ2, the expression level of NbFtsZ2 under mixotrophic condition suggests that NbFtsZ2 is enriched in the FtsZ ring formed before and after chloroplast division under mixotrophic condition.

The formation of FtsZ ring immediately after chloroplast division under mixotrophic condition suggests that the number of the FtsZ rings increase under certain conditions. The FtsZ ring formation in cells cultured in several concentrations of nitrogen, phosphorus, and glucose was therefore examined (Sumiya et al. 2012b). Under phosphate-enriched mixotrophic condition, multiple FtsZ rings are formed. A maximum of six FtsZ rings are formed just before chloroplast division; however, the chloroplast with multiple FtsZ rings does not divide at multiple sites simultaneously (Fig. 2; Sumiya et al. 2008). Transmission electron microscopy (TEM) analysis reveals that the cells cultured under phosphate-enriched mixotrophic condition contain only a single pair of PD rings (inner, middle, and outer PD rings) at the chloroplast division site (Sumiya et al. 2008), suggesting that the formation of the FtsZ and PD rings is induced via different mechanisms. The formation of multiple FtsZ rings is induced by reduplicated chloroplast DNA, as the amount of chloroplast DNA increases under phosphate-enriched mixotrophic condition and the inhibition of chloroplast DNA synthesis fails to induce multiple FtsZ ring formation (Fig. 2A; Sumiya et al. 2008). This reduplication of chloroplast DNA is in preparation for several chloroplast division without chloroplast
A

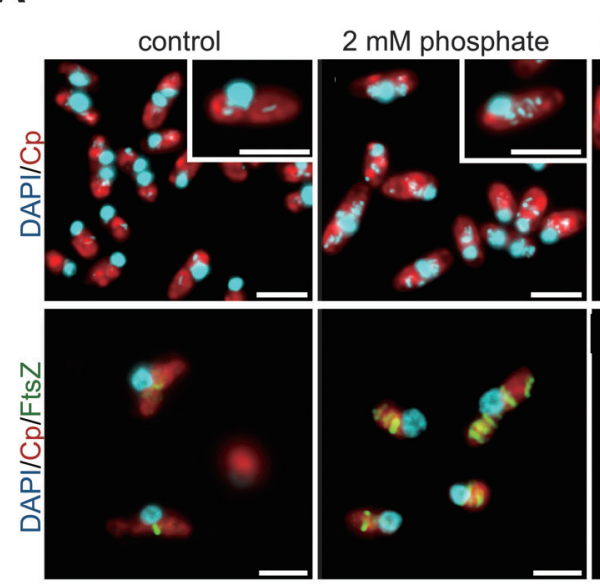

2 mM phosphate+ cpDNA syn inhibitor
B

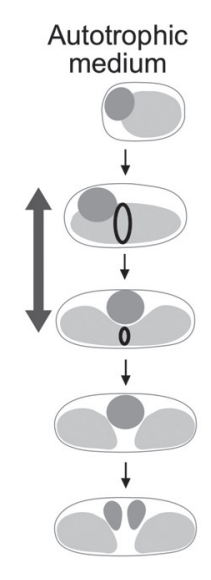

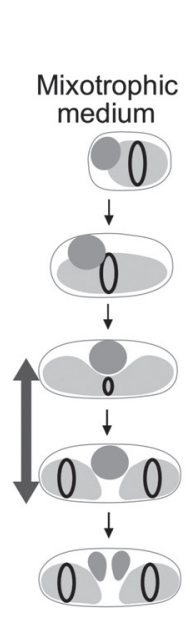

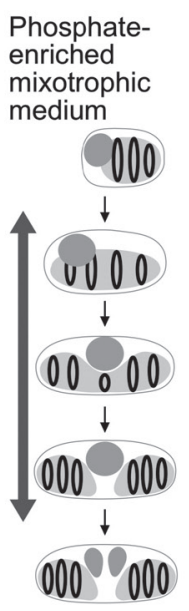

P-enriched medium to autotrophic medium

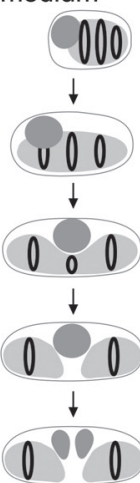

Figure 2 FtsZ ring formation during cell cycle under various conditions in the unicellular alga, Nannochloris bacillaris. (A) Immunofluorescence images of $N$. bacillaris cells cultured in phosphate-enriched mixotrophic medium with or without chloroplast DNA synthesis inhibitor. Cells were grown in the mixotrophic medium with poor-phosphate (control), phosphate-enriched mixotrophic medium ( 2 mM phosphate), or phosphate-enriched mixotrophic medium with fluorodeoxyuridine $(2 \mathrm{mM}$ phosphate + cpDNA syn inhibitor). Insets reveal enlarged images of DAPI-stained cells. Cyan $=$ DAPIstained DNA, green $=$ FtsZ, red $=$ autofluorescence of chlorophyll. Bars $=3 \mu \mathrm{m}$. (B) Schematic representation of FtsZ ring formation in various medium conditions. Dark gray and light gray indicate nuclei and chloroplasts, respectively. Black elliptical rings indicate FtsZ rings. Dark gray arrows indicate the predicted chloroplast DNA synthesis stage. Images were modified from Sumiya et al. (2008). 
DNA synthesis under poor nutrient condition. When $N$. bacillaris cells cultured under phosphate-enriched mixotrophic condition are transferred to the autotrophic condition, the amount of chloroplast DNA and the number of FtsZ rings decrease simultaneously without chloroplast DNA replication (Fig. 2B; Sumiya et al. 2012b). Chloroplast DNA is actively synthesized in the cells transferred from autotrophic to phosphate-enriched mixotrophic condition. In the land plants, the preferential synthesis of organelle DNA before multiple cell divisions has been reported (Suzuki et al. 1992, Fujie et al. 1993, Suzuki et al. 1995). Furthermore, the preferential synthesis of plastid DNA and the formation of multiple FtsZ rings have been observed in tobacco BY-2 cells (Momoyama et al. 2002). Thus, the formation of multiple FtsZ rings induced by reduplicated chloroplast DNA synthesis would be conserved from green algae to higher plants under certain conditions, although the FtsZ ring as well as the other components of the PD machinery are generally formed only once per chloroplast division cycle in unicellular algae.

\section{CHLOROPLAST DIVISION CHECKPOINT IN UNICELLULAR ALGAE}

In unicellular algae containing a single chloroplast, the chloroplast division machinery is assembled at least once per chloroplast division cycle to avoid the loss of chloroplast during cell division. The onset of chloroplast division is restricted to the $\mathrm{S}$ phase by S-phase-specific expression of the nuclear-encoded chloroplast division genes, and the chloroplast divides during the G2 phase (Fig. 3A; Miyagishima et al. 2012). In the case of the unicellular red alga, Cyanidioschyzon merolae, the FtsZ promoter contains a consensus binding site of S-phase-specific transcription factor E2F, suggesting that the timing of expression of the chloroplast division genes is determined at the transcriptional level (Miyagishima et al. 2014). In C. merolae cells arrested at $\mathrm{S}$ phase, the chloroplast division genes continue to be expressed, increasing the number of chloroplasts by 4-8 per cell (Itoh et al. 1996, Miyagishima et al. 2012). This suggests that the onset of chloroplast division is determined by the timing of expression of the chloroplast division genes. However, very little attention has been paid to the coordinate progression of the host cell cycle and chloroplast division after the onset of chloroplast division. To address this issue, the effect of blockage of chloroplast division on cell cycle progression has been examined in C. merolae, which contains a single chloroplast per cell. C. merolae is the useful organism for this study because the molecular mechanism of chloroplast division has been well understood in this alga, and a procedure for nuclear gene targeting has been developed (Kuroiwa et al. 1998, Minoda et al. 2004, Miyagishima 2011). Because the blockage of chloroplast division is likely to be lethal, an inducible gene expression system using the promoter of heat shock protein CMJ101C has been developed in C. merolae (Sumiya et al. 2014). The optimal temperature for the growth of C. merolae is $42^{\circ} \mathrm{C}$; in this system, a heat shock treatment of at least $30 \mathrm{~min}$ at $50^{\circ} \mathrm{C}$ is sufficient for inducing gene expression.

Dynamin is the founding member of a family of GTPases

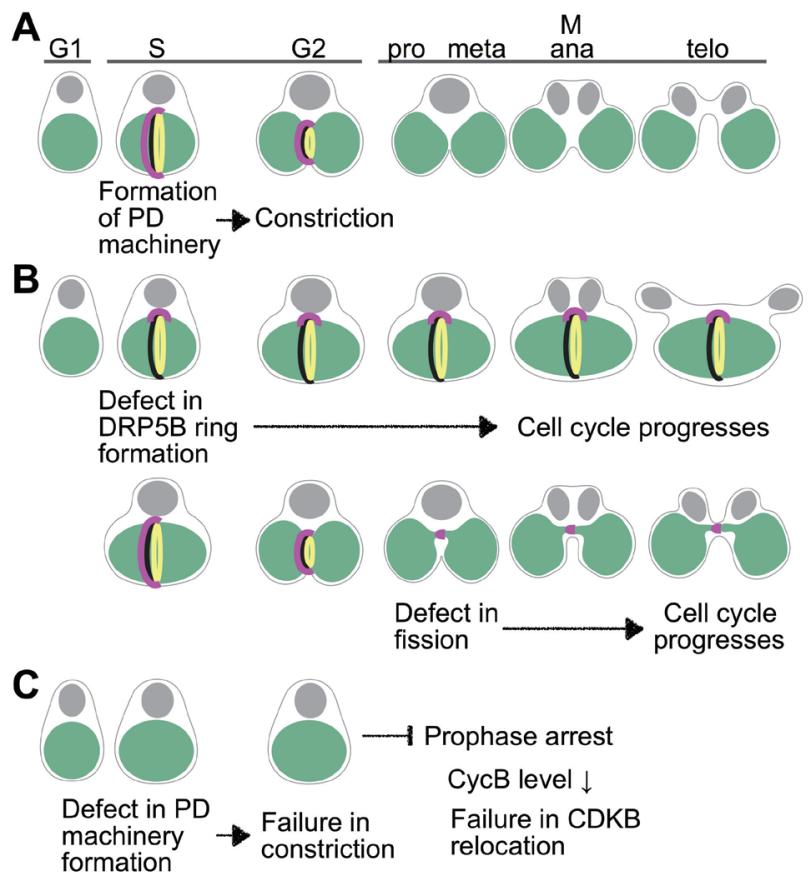

Figure 3 Schematic diagram revealing the effect of blockage of chloroplast division in C. merolae. (A) Formation of PD machinery comprising FtsZ (yellow), PDR1 (black), and DRP5B (magenta) during the $\mathrm{S}$ phase in wild type algae. The chloroplast divides during the G2 phase. (B) Expression of the dominant-negative form of DRP5B just before or during DRP5B ring formation hampers the constriction or fission of the chloroplast, whereas the cell cycle progresses. (C) Overexpression of FtsZ before the formation of PD machinery interferes with PD machinery formation, resulting in cell cycle arrest at prophase. Images were modified from Sumiya et al. (2016).

that plays a key role in endocytic membrane fission (Praefcke and McMahon 2004, Ferguson and De Camilli 2012). Mutations in the first GTP-binding consensus sequence of dynamin result in dominant-negative effects. DRP5B contains this consensus sequence, thus, the effect of dominant-negative DRP5B on chloroplast division has been examined in stably transformed C. merolae expressing GFP-DRP5B K135A under the control of heat shock promoter (Sumiya et al. 2014). The stable transformant cultured under continuous light is transferred to a dark condition to stop new entrance of G1-phase into S phase, then is heat-shocked twice at $50^{\circ} \mathrm{C} .24 \mathrm{~h}$ after transfer to the dark, two types of aberrant cells were observed. One is the two daughter cells connected by GFP-DRP5B K135A-positive tube-like structure. Each daughter cell contains a nucleus (Fig. 3B; Sumiya et al. 2014). The other is the cell which contains one spherical chloroplast and two nuclei (Fig. 3B; Sumiya et al. 2016). GFP-DRP5B K135A localizes as a dot at the nuclear side of the provisional chloroplast division site of the spherical chloroplast (Sumiya et al. 2016, Sumiya and Miyagishima 2017). Immunoblot analysis reveals that the $M$ phase marker is barely detectable $24 \mathrm{~h}$ after transfer to the dark (Sumiya et al. 2016). These results suggest that while the expression of dominant-negative DRP5B does not arrest cell cycle progression, it arrests the chloroplast division either at the onset of division site constriction or at the final fission stage. 
Overexpression of FtsZ impairs chloroplast division in the land plants (Stokes et al. 2000). To examine the effect of FtsZ overexpression on chloroplast division and cell-cycle progression, the heat-shock promoter and the Fts $Z$ ORF fusion are integrated into a C. merolae chromosomal locus (Sumiya et al. 2016). The transformant is synchronized under $12 \mathrm{~h}$ light/12 $\mathrm{h}$ dark photoperiod and then FtsZ is overexpressed during chloroplast division. In FtsZ-overexpressing cells, abnormal cells with a single chloroplast and two nuclei are observed, suggesting that the overexpression of Fts $Z$ during chloroplast division does not affect cell cycle progression. In contrast, overexpression of Fts $Z$ before the onset of chloroplast division impairs the chloroplast division and arrests cell cycle at prophase (Fig. 3C; Sumiya et al. 2016). While the overexpression of FtsZ before the onset of chloroplast division arrests the cell cycle at prophase, induction of dominant-negative DRP5B allows cell cycle progression. Induction of dominant-negative mutant of DRP5B induces slight constriction at the chloroplast division site but inhibits DRP5B ring formation (Sumiya and Miyagishima 2017). Collectively, these data suggest that the chloroplast division checkpoint of the host cell at prophase senses the onset of chloroplast constriction.

Cyclin B and cyclin-dependent kinase B (CDKB) regulate the transition of cells from $\mathrm{G} 2$ to $\mathrm{M}$ phase (Scofield et al. 2014). The CYCLIN B mRNA level is drastically reduced in FtsZ-overexpressing cells, whereas it peaked at $20 \mathrm{~h}$ in the control cells. The expression of $C D K B$ peaks at $16 \mathrm{~h}$ in the control cells and at $20 \mathrm{~h}$ in FtsZ-overexpressing cells. Immunofluorescence analysis reveals that cyclin B localizes to a few dots between the nucleus and chloroplast from the late $\mathrm{S}$ phase to anaphase in the control cells, but is absent from FtsZ-overexpressing cells. CDKB surrounds the nucleus in the late $\mathrm{S}$ phase, and then localizes to a few dots between the nucleus and chloroplast from the G2 phase to metaphase in the control cells. In contrast, CDKB remains localized around the nucleus in FtsZ-overexpressing cells (Sumiya et al. 2016). These results suggest that the dysfunction of cyclin B-CDKB complex induces prophase arrest in cells with blocked chloroplast division before the assembly of the PD machinery.

A similar phenomenon has been identified in the glaucophyte, Cyanophora paradoxa. The glaucophyte chloroplast, referred to as cyanelle, retains a peptidoglycan layer of cyanobacteria (Löffelhardt et al. 1997). In bacteria, proteins involved in the synthesis of proteoglycans bind to the cell division proteins, and septum formation requires peptidoglycan ingrowth (Typas et al. 2011). When cyanelle division is arrested before the division site constriction by the peptidoglycantargeted antibiotic carbenicillin, host cell cycle is arrested at a certain point before the anaphase. In contrast, when cyanelle division is arrested during the division site constriction by the septum formation inhibitor cephalexin, the nucleus divides into two nuclei, whereas cytokinesis is arrested during constriction by the undivided cyanelle (Sumiya et al. 2016). These results suggest that the chloroplast division checkpoint must have been established before the split of the glaucophyte from other algae.

\section{CONCLUSION AND REMARKS}

The mechanism of the coordinated cell and chloroplast division in unicellular algae has been elucidated through several studies. Chloroplast division is synchronized with host cell cycle by an interactive restriction (Fig. 4). First, the PD machinery is formed only during the $\mathrm{S}$ phase by the host cell (Miyagishima et al. 2012). The S-phase-specific expression or chloroplastdivision-phase-specific expression is reported in various algae except for FtsZ in the glaucophyte C. paradoxa (Gillard et al. 2008, Miyagishima et al. 2012). Among the components of PD machinery, the FtsZ ring formation is promoted by chloroplast DNA synthesis under certain culture conditions, such as high phosphate concentration (Sumiya et al. 2008). Second, the onset of chloroplast constriction releases the prophase arrest (Sumiya et al. 2016). Although DRP5B and the outer PD ring are lacked in C. paradoxa (Iino et al. 2003, Sato et al. 2009, Miyagishima

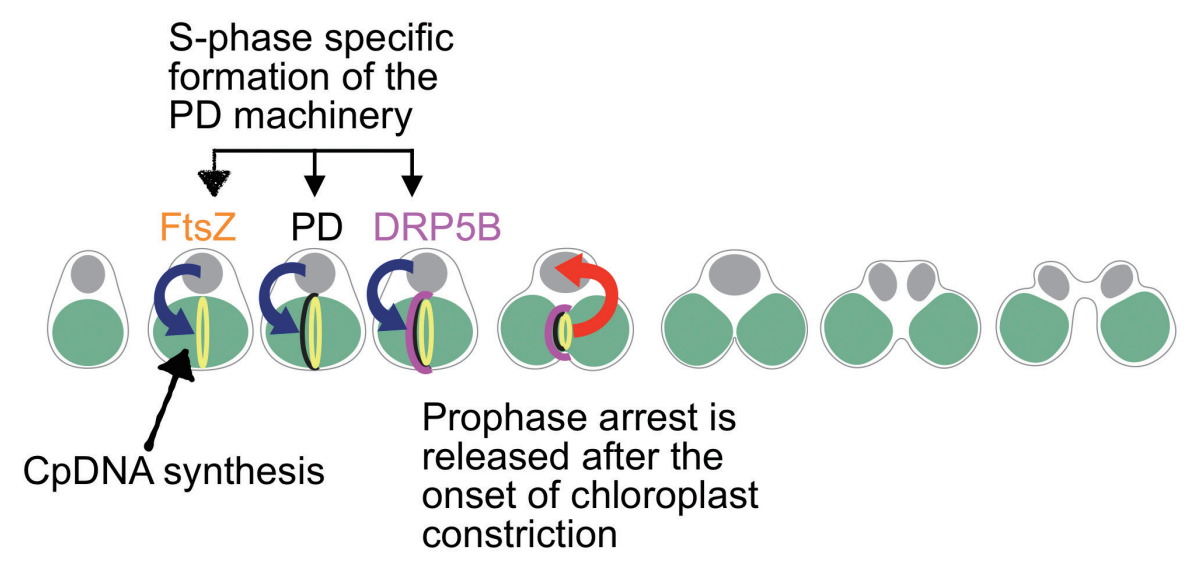

Figure 4 Schematic diagram revealing the coordinated division of cell and chloroplast in unicellular algae. The host cell restricts the onset of chloroplast division to the S phase by S-phase-specific expression of the chloroplast division proteins. In addition, the FtsZ ring formation is promoted by chloroplast DNA synthesis. The onset of chloroplast division releases the prophase arrest so that the host cell enters into metaphase upon chloroplast division. Images were modified from Sumiya et al. (2016). 
et al. 2012), the blockage of cyanelle division site constriction in C. paradoxa arrests cell cycle, thus, the mechanism of chloroplast division check point is achieved at a very early stage of the chloroplast acquisition.

Several aspects of the release from prophase arrest remain unclear. First, the detail of the stage of chloroplast division that is detected by the chloroplast division checkpoint is unclear. Our results suggest a certain stage between FtsZ ring formation and the recruitment of DRP5B is sensed by the host cell cycle. However, it still remains to be clarified at which stage of chloroplast division is sensed by the host cell cycle because only a few proteins have been identified as components of the PD machinery in the algae. Second, it is unknown whether cyclin B expression or CDKB relocalization is significant for prophase arrest. Identifying the trigger for prophase arrest would help us understand the mechanism used by the host cell to detect the block of chloroplast division. Third, the generality of this checkpoint remains poorly understood. Chloroplasts and cells divide asynchronously in the land plants, suggesting the loss of coordination between cell and chloroplast division in the land plants. Furthermore, it might not be necessary for algae with multiple chloroplasts per cell to control chloroplast division during the cell cycle. Further studies using various algae are needed to understand the establishment and the loss of coordinated cell and chloroplast division during the course of evolution from algae to land plants.

\section{ACKNOWLEDGMENTS}

The author would like to acknowledge the contributions to the following groups and individuals: Professor Shigeyuki Kawano and members in Kawano laboratory (The University of Tokyo) for the projects using Nannochloris bacillaris, and Professor Shin-ya Miyagishima and members in Miyagishima laboratory (National Institute of Genetics) for the project of chloroplast division checkpoint, which is selected for HIRASE award. This paper was supported by JSPS KAKENHI Grant Number JP17K18091.

\section{REFERENCES}

Arai, S., Takahashi, H., Takano, H., Sakai, A., and Kawano, S. (1998) Isolation, characterization, and chromosome mapping of an actin gene from the primitive green alga, Nannochloris bacillaris (Chlorophyceae). J Phycol 34: 477-485.

Bisson-Filho, A. W., Hsu, Y. P., Squyres, G. R., Kuru, E., Wu, F., Jukes, C., Sun, Y., Dekker, C., Holden, S., VanNieuwenhze, M. S., Brun, Y. V., and Garner, E. C. (2017) Treadmilling by FtsZ filaments drives peptidoglycan synthesis and bacterial cell division. Science 355: 739-743.

Chen, C., MacCready, J. S., Ducat, D. C., and Osteryoung, K. W. (2018) The molecular machinery of chloroplast division. Plant Physiol 176: 138-151.

Dorrell, R. G. and Howe, C. J. (2012) What makes a chloroplast? Reconstructing the establishment of photosynthetic symbioses. J Cell Sci 125: 1865-1875.

Erickson, H. P., Anderson, D. E., and Osawa, M. (2010) FtsZ in bacterial cytokinesis: cytoskeleton and force generator all in one. Microbiol Mol Biol Rev 74: 504-528.

Ferguson, S. M. and De Camilli, P. (2012) Dynamin, a membrane-remodelling GTPase. Nat Rev Mol Cell Biol 13: 75-88.

Fujie, M., Kuroiwa, H., Kawano, S., Mutoh, S., and Kuroiwa, T. (1993) Organelle DNA synthesis in the quiescent center of Arabidopsis thaliana (Col.). J Exp Bot 44: 689-693.

Gao, H., Kadirjan-Kalbach, D., Froehlich, J. E., and Osteryoung, K. W. (2003) ARC5, a cytosolic dynamin-like protein from plants, is part of the chloroplast division machinery. Proc Natl Acad Sci USA 100: 4328-4333.

Gillard, J., Devos, V., Huysman, M. J. J., De Veylder, L., D’Hondt, S., Martens, C., Vanormelingen, P., Vannerum, K., Sabbe, K., Chepurnov, V. A., Inzé, D., Vuylsteke, M., and Vyverman, W. (2008) Physiological and transcriptomic evidence for a close coupling between chloroplast ontogeny and cell cycle progression in the pennate diatom Seminavis robusta. Plant Physiol 148: 1394-1411.

Iino, M. and Hashimoto, H. (2003) Intermediate features of cyanelle division of Cyanophora paradoxa (Glaucocystophyta) between cyanobacterial and plastid division. $J$ Phycol 39:561-569.

Itoh, R., Takahashi, H., Toda, K., Kuroiwa, H., and Kuroiwa, T. (1996) Aphidicolin uncouples the chloroplast division cycle from the mitotic cycle in the unicellular red alga Cyanidioschyzon merolae. Eur J Cell Biol 71: 303-310.

Koide, T., Yamazaki, T., Yamamoto, M., Fujishita, M., Nomura, H., Moriyama, Y., Sumiya, N., Matsunaga, S., Sakamoto, W., and Kawano, S. (2004) Molecular divergence and characterization of two chloroplast division genes, Fts $Z 1$ and FtsZ2, in the unicellular green alga Nannochloris bacillaris (Chlorophyta). J Phycol 40: 546-556.

Kuroiwa, H., Mori, T., Takahara, M., Miyagishima, S. Y., and Kuroiwa, T. (2002) Chloroplast division machinery as revealed by immunofluorescence and electron microscopy. Planta 215: 185-190.

Kuroiwa, T., Kuroiwa, H., Sakai, A., Takahashi, H., Toda, K., and Itoh, R. (1998) The division apparatus of plastids and mitochondria. Int Rev Cytol 181: 1-41.

Löffelhardt, W., Bohnert, H. J., Bryant, D. A., and Hagemann, R. (1997) The cyanelles of Cyanophora paradoxa. Crit Rev Plant Sci 16: 393-413.

Minoda, A., Sakagami, R., Yagisawa, F., Kuroiwa, T., and Tanaka, K. (2004) Improvement of culture conditions and evidence for nuclear transformation by homologous recombination in a red alga, Cyanidioschyzon merolae 10D. Plant Cell Physiol 45: 667-671.

Miyagishima, S. Y., Itoh, R., Toda, K., Kuroiwa, H., and Kuroiwa, T. (1999) Real-time analyses of chloroplast and mitochondrial division and differences in the behavior of their dividing rings during contraction. Planta 207: 343-353.

Miyagishima, S. Y., Nishida, K., Mori, T., Matsuzaki, M., Higashiyama, T., Kuroiwa, H., and Kuroiwa, T. (2003) A plant-specific dynamin-related protein forms a ring at the 
chloroplast division site. Plant Cell 15: 655-665.

Miyagishima, S. Y. (2011) Mechanism of plastid division: From a bacterium to an organelle. Plant Physiol 155: 1533-1544.

Miyagishima, S. Y., Suzuki, K., Okazaki, K., and Kabeya, Y. (2012) Expression of the nucleus-encoded chloroplast division genes and proteins regulated by the algal cell cycle. Mol Biol Evol 29: 2957-2970.

Miyagishima, S. Y., Fujiwara, T., Sumiya, N., Hirooka, S., Nakano, A., Kabeya, Y., and Nakamura, M. (2014) Translation-independent circadian control of the cell cycle in a unicellular photosynthetic eukaryote. Nat Commun 5: 3807.

Momoyama, A., Miyazawa, Y., Miyagishima, S. Y., Mori, T., Misumi, O., Kuroiwa, H., and Kuroiwa, T. (2003). The division of pleomorphic plastids with multiple FtsZ rings in tobacco BY-2 cells. Eur J Cell Biol 82: 323-332.

Mori, T., Kuroiwa, H., Takahara, M., Miyagishima, S. Y., and Kuroiwa, T. (2001) Visualization of an FtsZ ring in chloroplasts of Lilium longiflorum leaves. Plant Cell Physiol 42: 555-559.

Okamoto, N. and Inouye, I. (2005) A Secondary Symbiosis in Progress? Science 310: 287-287.

Okamoto, N. and Inouye, I. (2006) Hatena arenicola gen. et sp. nov., a katablepharid undergoing probable plastid acquisition. Protist 157: 401-419.

Onuma, R. and Horiguchi, T. (2015) Kleptochloroplast enlargement, karyoklepty and the distribution of the cryptomonad nucleus in Nusuttodinium (=Gymnodinium) aeruginosum (Dinophyceae). Protist 166: 177-195.

Pedroza-Garcia, J.-A., Domenichini, S., Bergounioux, C., Benhamed, M., and Raynaud, C. (2016) Chloroplasts around the plant cell cycle. Curr Opin Plant Biol 34: 107-113.

Praefcke, G. J. and McMahon, H. T. (2004) The dynamin superfamily: universal membrane tubulation and fission molecules? Nat Rev Mol Cell Biol 5: 133-147.

Sato, M., Mogi, Y., Nishikawa, T., Miyamura, S., Nagumo, T., and Kawano, S. (2009) The dynamic surface of dividing cyanelles and ultrastructure of the region directly below the surface in Cyanophora paradoxa. Planta 229: 781-791.

Scofield, S., Jones, A., and Murray, J. A. H. (2014) The plant cell cycle in context. $J$ Exp Bot 65: 2557-2562.

Srinivasan, R., Mishra, M., Wu, L., Yin, Z., and Balasubramanian, M. (2008) The bacterial cell division protein FtsZ assembles into cytoplasmic rings in fission yeast. Genes Dev 22: 17411746.

Stokes, K. D., McAndrew, R. S., Figueroa, R., Vitha, S., and Osteryoung, K. W. (2000) Chloroplast division and morphology are differentially affected by overexpression of FtsZ1 and FtsZ2 genes in Arabidopsis. Plant Physiol 124: 1668-1677.

Sumiya, N., Hirata, A., and Kawano, S. (2008) Multiple FtsZ rig formation and reduplicated chloroplast DNA in Nannochloris bacillaris (Chlorophyta, Trebouxiophyceae) under phosphate-enriched culture. J Phycol 44: 1476-1489.

Sumiya, N., Yamazaki, T., Owari, S., Yamamoto, M., Watanabe,
K., and Kawano, S. (2012a) Chloroplast division and differentially regulated expression of FtsZ1 and FtsZ2 in the synchronous culture of Nannochloris bacillaris (Chlorophyta, Trebouxiophyceae). Cytologia 77: 59-66.

Sumiya, N., Owari, S., Watanabe, K., and Kawano, S. (2012b) Role of multiple FtsZ rings in chloroplast division under oligotrophic and eutrophic conditions in the unicellular green alga Nannochloris bacillaris (Chlorophyta, Trebouxiophyceae). J Phycol 48: 1187-1196.

Sumiya, N., Fujiwara, T., Kobayashi, Y., Misumi, O., and Miyagishima, S. Y. (2014) Development of a heat-shock inducible gene expression system in the red alga Cyanidioschyzon merolae. PLoS ONE 9: e111261.

Sumiya, N., Fujiwara, T., Era, A., and Miyagishima, S. Y. (2016) Chloroplast division checkpoint in eukaryotic algae. Proc Natl Acad Sci USA 113: E7629-E7638.

Sumiya, N. and Miyagishima, S. Y. (2017) Hierarchal order in the formation of chloroplast division machinery in the red alga Cyanidioschyzon merolae. Commun Integr Biol 10: e1294298.

Suzuki, T., Kawano, S., Sakai, A., Fujie, M., Kuroiwa, H., Nakamura, H., and Kuroiwa, T. (1992) Preferential mitochondrial and plastid DNA synthesis before multiple cell divisions in Nicotiana tabacum. J Cell Sci 103: 831-837.

Suzuki, T., Sasaki, N., Sakai, A., Kawano, S., and Kuroiwa, T. (1995) Localization of organelle DNA synthesis within the root apical meristem of rice. J Exp Bot 282: 19-25.

Typas, A., Banzhaf, M., Gross, C. A., and Vollmer, W. (2011) From the regulation of peptidoglycan synthesis to bacterial growth and morphology. Nat Rev Micro 10: 123-136.

Vitha, S., McAndrew, R., and Osteryoung, K. (2001) FtsZ ring formation at the chloroplast division site in plants. $J$ Cell Biol 153: 111-119.

Yamamoto, M., Nozaki, H., and Kawano, S. (2001) Evolutionary relationships among multiple modes of cell division in the genus Nannochloris (Chlorophyta) revealed by genome size, actin gene multiplicity, and phylogeny. J Phycol 37: 106120.

Yamamoto, M., Nozaki, H., Miyazawa, Y., Koide, T., and Kawano, S. (2003) Relationship between presence of a mother cell wall and speciation in the unicellular microalga Nannochloris (Chlorophyta). J Phycol 39: 172-184.

Yamamoto, M., Nishikawa, T., Kajitani, H., and Kawano, S. (2007) Patterns of asexual reproduction in Nannochloris bacillaris and Marvania geminata (Chlorophyta, Treouxiophyceae). Planta 226: 917-927.

Yang, X., Lyu, Z., Miguel, A., McQuillen, R., Huang, K. C., and Xiao, J. (2017) GTPase activity-coupled treadmilling of the bacterial tubulin FtsZ organizes septal cell wall synthesis. Science 355: 744-747.

Yoshida, Y., Kuroiwa, H., Misumi, O., Nishida, K., Yagisawa, F., Fujiwara, T., Nanamiya, H., Kawamura, F., and Kuroiwa, T. (2006) Isolated chloroplast division machinery can actively constrict after stretching. Science 313: 1435-1438.

Yoshida, Y., Kuroiwa, H., Misumi, O., Yoshida, M., Ohnuma, 
M., Fujiwara, T., Yagisawa, F., Hirooka, S., Imoto, Y., Matsushita, K., Kawano, S., and Kuroiwa, T. (2010) Chloroplasts divide by contraction of a bundle of nanofilaments consisting of polyglucan. Science 329: 949953.

Received: 5 February 2018 / Accepted: 16 February 2018 